\title{
模型跟随大系统的稳定性分析与 时变加权和方法
}

\author{
徐肖豪** 黄文虎 吴罯华 \\ (哈尔浣工业大学空间科学系,哈尔摈 150006)
}

关调大系统、稳定性、Lyapunov 方法

\section{一、问题的提出}

以往大系统的稳定性问题, 主要是研究形如

$$
\frac{d x_{i}}{d t}=\phi_{i}\left(x_{i}, t\right)+p_{i}(X, \imath), \quad x_{i} \in R^{m_{i}}, i=1, \cdots, N
$$

的复合系统的零解 $X=0$ 的稳定性, 其中 $X^{T}-\left[x_{1}^{1}, \cdots, x_{N}^{T}\right]$. 对此, 刘永清 ${ }^{(1)}$ 在 1959 年 即根据秦元勋的稳定性分解思想, 提出了大系统的 Lyapunov 函数分解法, 并由此得到了许多 重要的分析结果. 随后, Thompson ${ }^{[2]}$ 用各子系统 Lyapunov 函数的加权和来构造标量 Lyapunov 函数, 导得了大系统全局指数稳定的条件. 这一方法即称为加权和方法. Araki ${ }^{[3]}$ 将加权和方法与 $M$-矩阵概念相结合, 研究了复合系统稳定的条件. 在加权和方法中, Lyapunov 函数的形式为

$$
V(\cdot)-\sum_{i=1}^{i} d_{i} v_{i}(\cdot),
$$

其中 $v_{i}(\cdot)$ 为子系统的 Lyapunov 函数, $N$ 为子系统的个数, $d_{i}>0$ 为加权因子. 在以往的 研究中, 均取 $d_{i}$ 为常数, 因而我们称其为定常加权和方法.

在控制工程中, 常遇到另一类型的大系统一模型跟随大系统. 此时需考察系统 (1.1)式 跟随给定参考模型

$$
\frac{d y_{i}}{d t}=\psi_{i}\left(y_{i}, t\right)+q_{i}(Y, t), \quad y_{i} \in R^{m_{i}, i}-1, \cdots, N
$$

的动态特性, 其中 $Y^{T}=\left[y_{1}^{T}, \cdots, y_{N}^{T}\right]$. 若记 $e_{i}-x_{i}-y_{i}, i-1, \cdots, N$, 则它等价于研究 复合误差系统

$$
\frac{d e_{i}}{d t}=f_{i}\left(e_{i}, x_{i}, t\right)+g_{i}(e, X, t), \quad i-1, \cdots, N
$$

的平衡点 $e-0$ 的稳定性, 其中 $e^{I}-\left[e_{1}^{T}, \cdots, e_{N}^{T}\right], f_{i}\left(e_{i}, x_{i}, t\right)-\phi_{i}\left(x_{i}, t\right)-\psi_{i}\left(y_{i}, t\right)$, $g_{i}(e, X, t)-p_{i}(X, t)-q_{i}(Y, t)$, 且已假设成立 $f_{i}\left(0, x_{i}, t\right)-0, g_{i}(0, X, t)-0$. 系统 (1.4)式可看成由孤立子系统

本文 1989 年1月12 日收到. 1989 年9月25日收到修改稿.

- 国家自然科学基金资助项目.

** 现工作单位: 南京航空学院自动战制系. 


$$
\frac{d e_{i}}{d t}-f_{i}\left(e_{i}, x_{i}, z\right), \quad i-1, \cdots, N
$$

经关联项

$$
z_{i}=g_{i}(e, X, t), \quad i-1, \cdots, N
$$

连接而成的复合系统. 由于当 $y_{i} \equiv 0, i-1, \cdots, N$ 时, (1.4)式即为通常研究的(1.1)式, 因 此模型跟随大系统显然有更为普遍性的意义.

虽然在模型参考自适应系统的研究中, 已经应用了超稳定性等原理来保证控制系统的稳 定性, 但对形如 (1.4)式的模型跟随大系统, 迄今尚无类似(1.1)式系统的判稳准则.这是由于以 往研究的大系统 (1.1)式, 需考察 $X^{T} A X$ 形式的二次型, 它的正定性易于判别; 而模型跟随大 系统涉及的是形如 $X^{T} A Y$ 的准二次型, 此时即使 $A$ 为正定或 $M$-矩阵, $X 、 Y$ 均为正矢量, 也 不易确定其正定性, 因而若采用定常加权和方法, 则很难得到一般性的结果. 为此, 我们提出 了时变加权和方法, 即取

$$
V(\cdot)-\sum_{i=1}^{N} d_{i}(t) v_{i}(\cdot)
$$

为预选 Lyapunov 函数, 其中 $d_{i}(t)>0$ 为时变权因子. 它是以往定常加权和方法的推广和 发展. 基于这一方法, 导得了判别模型跟随大系统稳定性的一般准则, 从而推广了大系统定性 分析的现有成果.

\section{二、主熼结果}

定理 1 复合系统 (1.4)式的平衡点 $e-0$ 是大范围一致渐近稳定的, 如果以下条件得 以满足:

(i) 对每个孤立子系统 (1.5) 式, 存在一个正定、递降、径向无界且连续可微的函数 $v_{i}\left(e_{i}, t\right)$ 使

$$
\begin{aligned}
& \left.\frac{d v_{i}}{d t}\right|_{(1.5)} \leqslant-\alpha_{i} u_{i}^{2}\left(e_{i}\right)-\beta_{i} u_{i}\left(e_{i}\right) w_{i}\left(x_{i}\right), \\
& \left|\operatorname{grad}_{i} v_{i}\right| \leqslant u_{i}\left(e_{i}\right)
\end{aligned}
$$

成立, 其中 $\alpha_{i} 、 \beta_{i}$ 均为正常数, $u_{i}\left(e_{i}\right)$ 为正定函数, $w_{i}\left(x_{i}\right)$ 为非负定函数.

(ii) 存在常数 $\gamma_{i j}$, 使得

$$
\left|g_{i}(e, X, t)\right| \leqslant \sum_{i=1}^{N} \gamma_{i j} w_{i}\left(x_{i}\right),
$$

且对 $i+j$, 有 $\gamma_{i j} \geqslant 0$.

(iii) 矩阵 $H-\left(h_{i i}\right) \in R^{N \times N}$ 为 $M$-矩阵,

(iv) 函数 $n_{i}\left(e_{i}\right)$ 满足关系式:

$$
h_{i i}-\beta_{i}-\gamma_{i i}, \quad h_{i j}--\gamma_{i j}(i \neq j) .
$$

$$
\dot{u}_{i}\left(e_{i}\right)>-\alpha_{i} u_{i}^{3}\left(e_{i}\right) / v_{i}\left(e_{i}, t\right) \text {. }
$$

证 Lyapunov 预选函数 $V(t)$ 取如(1.7)式, 其中时变权因子 $d_{i}(t)>0$ 满足

$$
d_{i}(t) u_{i}\left(e_{i}\right)-k_{i}, i-1, \cdots, N,
$$

$k_{i}$ 为一正常数. 因此, $V(t)$ 为一个正定、递降和径向无界的函数.

$$
\text { 由(2.1)-(2.3)式, 有 }
$$




$$
\begin{aligned}
\sum_{i=1}^{N} d_{i}(t) \dot{v}_{i}\left(e_{i}, t\right)- & \sum_{i=1}^{N} d_{i}(t)\left\{\left.\frac{d v_{i}}{d t}\right|_{(1.5)}+\left(\operatorname{grad}_{i} v_{i}\right)^{T} g_{i}(e, X, t)\right\} \\
< & \sum_{i=1}^{N} d_{i}(t)\left\{-\alpha_{i} u_{i}^{2}\left(e_{i}\right)-\beta_{i} u_{i}\left(e_{i}\right) w_{i}\left(x_{i}\right)\right. \\
& \left.+u_{i}\left(e_{i}\right) \sum_{i=1}^{N} \gamma_{i j} w_{j}\left(x_{i}\right)\right\} \\
& <\sum_{i=1}^{N}-d_{i}(t) u_{i}\left(e_{i}\right)\left\{\left(\beta_{i}-\gamma_{i i}\right) w_{i}\left(x_{i}\right)-\sum_{i \neq i}^{N} \gamma_{i} w_{i}\left(x_{i}\right)\right\} . \\
& =-B^{T} H W,
\end{aligned}
$$

其中 $B^{T}-\left[d_{1}(t) u_{1}\left(e_{1}\right), \cdots, d_{N}(t) u_{N}\left(e_{N}\right)\right], W^{T}-\left[w_{1}\left(x_{1}\right), \cdots, w_{N}\left(x_{N}\right)\right]$.

因 $H$ 为 $M$-矩阵, 则必存在实向量 $K=\left[k_{1}, \cdots, k_{N}\right]^{T}, C-\left[c_{1}, \cdots, c_{N}\right]^{T}, k_{i}>0$, $c_{i}>0$, 使

$$
H^{T} K=C
$$

成立, 因而可取 $d_{i}(t)$ 满足(2.6)式,则知

$$
\sum_{i=1}^{N} d_{i}(t) \nu_{i}\left(e_{i}, t\right)<-C^{T} W=-\sum_{i=1}^{N} c_{i} w_{i}\left(x_{i}\right) \leqslant 0 .
$$

由(2.6)式,有

$$
d_{i}(t) u_{i}\left(e_{i}\right)=-d_{i}(t) \dot{u}_{i}\left(e_{i}\right)
$$

则

$$
\begin{aligned}
V(t) & =\sum_{i=1}^{N}\left\{d_{i}(t) \dot{v}_{i}\left(e_{i}, t\right)-d_{i}(t) u_{i}^{-1}\left(e_{i}\right) v_{i}\left(e_{i}, t\right) \dot{u}_{i}\left(e_{i}\right)\right\} \\
& \leqslant \sum_{i=1}^{N}\left\{-d_{i}(t)\left[\alpha_{i} u_{i}^{2}\left(e_{i}\right)+u_{i}^{-1}\left(e_{i}\right) v_{i}\left(e_{i}, t\right) \dot{u}_{i}\left(e_{i}\right)\right]-c_{i} w_{i}\left(x_{i}\right)\right\} .
\end{aligned}
$$

当 (2.5) 式成立时, 显见 $V(t)<0$, 因而复合系统 $(1.4)$ 式的平衡点 $e-0$ 是大范围一致渐近 稳定的.

定理 2 复合系统(1.4)式的平衡点 $e=0$ 是大范围一致渐近稳定的, 如果以下条件得 以满足:

(i) 对每个孤立子系统 (1.5)式, 存在一个正定、递降、径向无界且连续可微的函数 $v_{i}\left(e_{i}\right.$, ヶ), 使

$$
\left.\frac{d v_{i}}{d t}\right|_{(1.5)} \leqslant-\alpha_{i} u_{i}^{2}\left(e_{i}\right)-\beta_{i} u_{i}\left(e_{i}\right) w_{i}\left(x_{i}\right)
$$

成立, 其中 $\alpha_{i}>0, \beta_{i}$ 为一常数, $\mu_{i}\left(e_{i}\right)$ 为正定函数, 而 $w_{i}\left(x_{i}\right)$ 为非负定函数.

(ii) 存在常数 $\gamma_{i j}$, 使得

$$
\left(\operatorname{grad}_{i} \nu_{i}\right)^{T} g_{i}(e, X, t) \leqslant u_{i}\left(e_{i}\right) \sum_{i=1}^{N} \gamma_{i i} w_{i}\left(x_{i}\right),
$$

且对 $i \neq j, r_{i j} \geqslant 0$.

(iii) 同定理 1 的条件 (iii) 与 (iv).

定理 3 复合系统(1.4)的平衡点 $e=0$ 是大范围一致渐近稳定的, 如果以下条件得以 


\section{满足:}

(i) 对 $\sigma_{i}>0$, 成立定理 2 的条件(i).

(ii) 存在常数 $r_{i i} \geqslant 0$, 使得

$$
\left(\operatorname{grad}_{i} \nu_{i}\right)^{T} g_{i}(e, X, t) \leqslant u_{i}\left(e_{i}\right)\left[\sum_{i=1}^{N} \gamma_{i j} u_{i}\left(e_{i}\right) w_{i}\left(x_{i}\right)\right]^{\frac{1}{2}} \text {. }
$$

(iii) 矩阵 $H^{\prime}-\left(h_{i j}^{\prime}\right) \in R^{N \times N}$ 为 $M$-矩阵.

$$
h_{i i}^{\prime}-\beta_{i}-\frac{\gamma_{i j}}{2 \alpha_{i}}, \quad h_{i j}^{\prime}--\frac{\gamma_{i j}}{2 \alpha_{i}} \quad(i \neq j) .
$$

(iv) 函数 $n_{i}\left(e_{i}\right)$ 满足下式:

$$
2 \dot{u}_{i}\left(e_{i}\right)>-\alpha_{i} u_{j}^{3}\left(e_{i}\right) / v_{i}\left(e_{i}, z\right) .
$$

定理 2、3 的证明从略. 注意到定理 2 中由于 $\beta_{i}$ 可以为负值,因而允许始立子系统为不稳 定的.

\section{考文翰}

-11]刘永清、来中昆,大型动力系统的理论与应用,华南工学院出版社, 广州, 1988 .

\{2\} Thompson, W.E., IEEE Trans. Antom. Contr., 15(1970), 504-506.

(3) Araki, M., IEEE Trans. Autom. Contr., 23(1978), 129-142. 